\title{
Ionospheric perturbations in possible association with the 2010 Haiti earthquake, as based on medium-distance subionospheric VLF propagation data
}

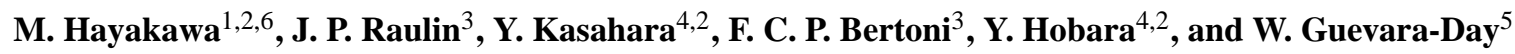 \\ ${ }^{1}$ The University of Electro-Communications (UEC), Advanced Wireless Communications Research Center, \\ 1-5-1 Chofugaoka, Chofu Tokyo 182-8585, Japan \\ ${ }^{2}$ UEC, Research Station on Seismo Electromagnetics, Chofu Tokyo, Japan \\ ${ }^{3}$ Centro de Radio Astronomia e Astrofisica, Universidade Presbiteriana Mackenzie, Escola de Engenharia, São Paulo, Brazil \\ ${ }^{4}$ UEC, Graduate School of Informatics and Electronic Engineering, Chofu Tokyo, Japan \\ ${ }^{5}$ Comisión Nacional de Investigación y Desarrollo Aeroespacial (CONIDA), Lima, Peru \\ ${ }^{6}$ Information Systems Inc., Earthquake Analysis Laboratory, 4-8-15-405 Minami-Aoyama, Minato-ku, \\ Tokyo 107-0062, Japan
}

Received: 26 October 2010 - Accepted: 18 November 2010 - Published: 16 February 2011

\begin{abstract}
Ionospheric perturbations in possible association with the 2010 Haiti earthquake occurred on 12 January 2010 (with a magnitude of 7.0 and depth of $10 \mathrm{~km}$ ) are investigated on the basis of subionospheric propagation data from the NAA transmitter on the east coast of the USA to a VLF receiving station in Peru. The local nighttime VLF amplitude data are extensively investigated during the period from the beginning of October 2009 to the end of March 2010, in which the trend (nighttime average amplitude), dispersion and nighttime fluctuation are analysed. It is found that a clear precursory ionosphere perturbation is detected just around New Years day of 2010, about 12 days before the main shock, which is characterised by the simultaneous decrease in the trend and the increases in dispersion and nighttime fluctuation. An additional finding might be the presence of the effect of the Earth's tide one and two months before the main shock, which can only be seen for a huge EQ.
\end{abstract}

\section{Introduction}

Since the clear evidence of the presence of ionospheric perturbations for the disastrous 1995 Kobe earthquake (EQ) by means of subionospheric VLF/LF propagation data (Hayakawa et al., 1996), there have been accumulated a substantial number of VLF/LF subionospheric evidences on seismo-ionospheric perturbations (see the recent reviews by Hayakawa, 2007, 2009; Hayakawa and Hobara, 2010).

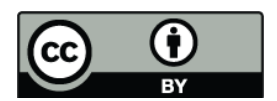

Correspondence to: M. Hayakawa (hayakawa@whistler.ee.uec.ac.jp)
These VLF/LF signals have been extensively utilized to investigate the perturbations in the lower ionosphere (i.e., D/E layers) due to seismic activities and there have been two different ways of investigation performed; (i) case studies (detailed studies for any particular huge EQ), and (ii) statistical studies on the correlation between the ionospheric perturbations and EQs.

As for case studies, we can list several huge EQs, including Japanese EQs, (1) Tokachi-oki EQ (25 September 2003; $M=8.3$ ) (Shvets et al., 2004), (2) Niigata-Chuetsu EQ (23 October 2004; $M=6.8$ ) (Hayakawa et al., 2006; Yamauchi et al., 2007), (3) an EQ in Taiwan, Chi-chi EQ (20 September 1999; $M=7.6$ ) (Ohta et al., 2002; Hayakawa et al., 2005), (4) an extremely huge EQ in Indonesia, Sumatra EQ (26 December 2004; $M=9.0$ ) (Horie et al., 2007a, b) and also (5) an Italian EQ, L'Aquila EQ (6 April 2009; $M=6.3$ ) (Rozhnoi et al., 2009; Biagi et al., 2009). These case studies have enabled us to investigate extensively the spatial and temporal characteristics of seismo-ionospheric perturbations. Together with the corresponding satellite observations by DEMETER, the detailed spatial properties of seismo-ionospheric perturbations in the case of the 2004 Sumatra EQ were studied (Molchanov et al., 2006) and we will be able to study the mechanism of seimo-ionospheric perturbations, in other words, the lithosphere-atmosphereionosphere coupling.

At the same time, statistical studies should be performed in order to convince the community that ionospheric perturbations really take place in association with EQs. Unfortunately, there are very few reports on this statistical

Published by Copernicus Publications on behalf of the European Geosciences Union. 
correlation between VLF/LF propagation anomalies and EQs. Rozhnoi et al. (2004) studied one propagation path, but for a period of 2 years and Maekawa et al. (2006) studied again one particular propagation path for 6 years. Then, Kasahara et al. (2008) have largely extended the period of analysis up to 7 years and they have also used different paths. The conclusion claimed by these authors is that ionospheric perturbations by means of the subionospheric VLF/LF data tend to definitely take place before an EQ when the EQ magnitude is greater than 5.5 and when the depth is shallow $(d<40 \mathrm{~km})$. Also, a statistical study using an Indian transmitter has been done by Chakrabarti et al. (2010), who have shown a possible correlation with EQs. Recently, two papers have been published about the rather complete statistical correlation between the ionospheric perturbation and EQs over nine years by using different VLF/LF propagation paths (Hayakawa et al., 2010a, b). The authors conclude that the trend (average nighttime amplitude) shows a definite decrease exceeding $2 \sigma$ ( $\sigma$ : standard deviation) and the dispersion and nighttime fluctuation show an increase exceeding $2 \sigma$. This significant statistical correlation indicates the definite occurrence of seismo-ionospheric perturbations several days before an EQ.

The purpose of this paper is to report on the detailed propagation anomalies in possible association with the disastrous 2010 Haiti EQ by using a medium-distance propagation path. The spatial and temporal properties of seismo-ionospheric perturbations are discussed extensively in the context of our previous case studies and our recent statistical studies. Finally, some comments on the generation mechanism of seismo-ionospheric perturbations are given.

\section{The 2010 Haiti EQ}

The 2010 Haiti EQ took place at the geographic coordinates $\left(18.45^{\circ} \mathrm{N}, 72.45^{\circ} \mathrm{W}\right)$ at $21: 53: 09 \mathrm{UTC}$ on 12 January 2010 (LT = 16:53). The magnitude of this EQ was $7.0\left(M_{\mathrm{w}}\right.$, moment magnitude) and the depth was $10 \mathrm{~km}$, these conditions being extremely favourable for inducing ionospheric perturbations (e.g., Hayakawa et al., 2010a). The epicentre was located about $15 \mathrm{~km}$ west-south-west of the capital of Port-France of the Republic of Haiti. This area is characterised by the proximity to the collisional boundary between the North American and Caribbean plates, where we expect very often shallow, inland EQs. The fault responsible for this EQ is considered to be one in the Enriquillo-Plaintain Garden fault system. Two significant aftershocks took place; the first one occurred at 17:00 on 12 January (LT) just after the main shock and another at 06:03 LT on 20 January (about 8 days after the main shock). Both aftershocks had a magnitude of 5.9. There were reports of many casualties caused by this EQ; 220000 people died and 320000 people were injured.

\section{VLF subionospheric propagation and propagation anomaly analyses}

In order to find any seismogenic effects in subionospheric VLF/LF propagation data, there have been developments on two characteristic ways of data analysis. The first is called "terminator time" method, in which we trace the temporal change of the time of terminator (sunrise and sunset). This method was initially proposed for the Kobe EQ (Hayakawa et al., 1996; Molchanov et al., 1998), which is currently and effectively used for short-distance (less than a few $\mathrm{Mm}$ ) propagation path. An alternative technique is called "nighttime fluctuation method", in which we utilize the data only during the local nighttime. After estimating the difference of $A(t)$ (amplitude $A$ at a time $t$ on a particular day) from $\langle A(t)\rangle$ (the average amplitude at the same time $t$ over \pm 15 days around the current day), we measure the following physical parameters (1) nighttime average amplitude or trend, (2) dispersion, and (3) nighttime fluctuation (as defined by the negative $d A(t)<0$ integrated over the local). Then, in the view of previous results, when we have a propagation anomaly, the average amplitude (trend) is found to decrease significantly and both the dispersion and nighttime fluctuation parameters are known to be enhanced statistically significantly (Hayakawa et al., 2010a).

These two methods are currently used for short-distance (less than $\sim 2 \mathrm{Mm}$ ) propagation paths and it is found that both methods are equally effective for this distance. However, the terminator time method is not found to be so effective for medium-distance $(6-8 \mathrm{Mm})$ propagation paths and for North-South oriented propagation paths (Maekawa and Hayakawa, 2006). On the other hand, it was recently found that the nighttime fluctuation method works well for medium-distance propagation paths (Horie et al., 2007a, b; Kasahara et al., 2010). For example, Kasahara et al. (2010) have used the propagation paths from the Australian NWC transmitter to Japanese receiving stations (6-8 Mm) to search for ionospheric perturbations associated with EQs in Asia, for which the nighttime fluctuation method has been found to be extremely useful in finding the EQ signature.

First of all, we have to indicate the relative location of the EQ epicentre and our VLF propagation path. We have established a VLF/LF network in South America (socalled SAVNET, Raulin et al., 2009), and a few possible combinations of the VLF transmitter and receiving point, are available for this Haiti EQ. Figure 1 illustrates the VLF transmitter, NAA (frequency $=24.0 \mathrm{kHz}$ ) located on the East coast of the USA (Cutler, geographic coordinates: $44.39^{\circ} \mathrm{N}$, $76.12^{\circ} \mathrm{W}$ ) and our VLF receiving station of PLO (Punta Lobos, $12.50^{\circ} \mathrm{S}, 76.80^{\circ} \mathrm{W}$, about $60 \mathrm{~km}$ south of Lima) in Peru. The great-circle path of this transmitter-receiver combination and also its corresponding 5th Fresnel zone are plotted as well in Fig. 1. The EQ epicentre is found to be located exactly within the wave sensitive area and 
we would expect significant EQ signatures within the VLF dataset. The VLF/LF receiving system, as described in Raulin et al. (2009), is quite similar to that used for the Japanese network (Hayakawa, 2010a), and the data sampling is $1 \mathrm{~s}$.

Based on the discussion above, we will use the nighttime fluctuation method for the analysis of this Haiti EQ. The propagation distance from the transmitter, NAA, to our VLF/LF station of PLO is $6.46 \mathrm{Mm}$, which is found to be comparable to that used in Kasahara et al. (2010). First we choose the nighttime period between UT $=0-6 \mathrm{~h}(\mathrm{LT}=\mathrm{UT}-$ $3 \mathrm{~h}$ ) as local nighttime for this propagation path. As we have done in our previous papers (Kasahara et al., 2010; Hayakawa et al., 2010a), we use a residue $d A(t)$ as defined by $A(t)-<A(t)>$ where $A(t)$ is the amplitude at a time $t$ on a particular day (this is the same as before), while $\langle A(t)\rangle$ is the average amplitude at the same $t$ over the previous 30 days before the current day $(-30 \sim-1$ day of the current day, unlike the previous \pm 15 days). This procedure enables us to exclude the long-term variation such as the seasonal effect and to make clear the short-term variation such as seismogenic effect. Then, we estimate the following three physical parameters: (1) trend (average amplitude during our local nighttime), (2) dispersion (conventional statistical quantity), and (3) NF (nighttime fluctuation) as the temporal integration of $d A(t)<0$ parts during the local night. These quantities are normalized by their corresponding standard deviations.

\section{VLF analysis results}

Figure 2 depicts the obtained temporal evolutions of these three physical parameters (from top, trend, dispersion and NF). Grey parts indicate the periods of lack of observation due to maintenance or failure in the system. We have analysed data from the beginning of October 2009 to the beginning of April 2010 (seven months) as a preliminary analysis. Instead of looking at the temporal variations of each physical parameter separately, it is more appropriate to look at them globally. Our previous work by Kasahara et al. (2010) for medium-distance propagation paths (nearly the same distance as in the present Haiti case) indicates that the VLF/LF anomaly is characterised by a significant decrease in trend along with simultaneous increases in dispersion and $\mathrm{NF}$, as it was found for short-distance propagation cases (Hayakawa et al., 2010a, b). Therefore, it is better to pay attention to the global variations of the three parameters as a pattern. When we look at Fig. 2 in such a way, we can notice a few time periods, marked using green symbols, during which the trend as the most important parameter is depleted exceeding its $2 \sigma$ ( $\sigma$ : standard deviation) criterion and both dispersion and NF are enhanced by exceeding or approaching their corresponding $2 \sigma$ criteria. It is not important to have these properties exactly on the same day, but during the

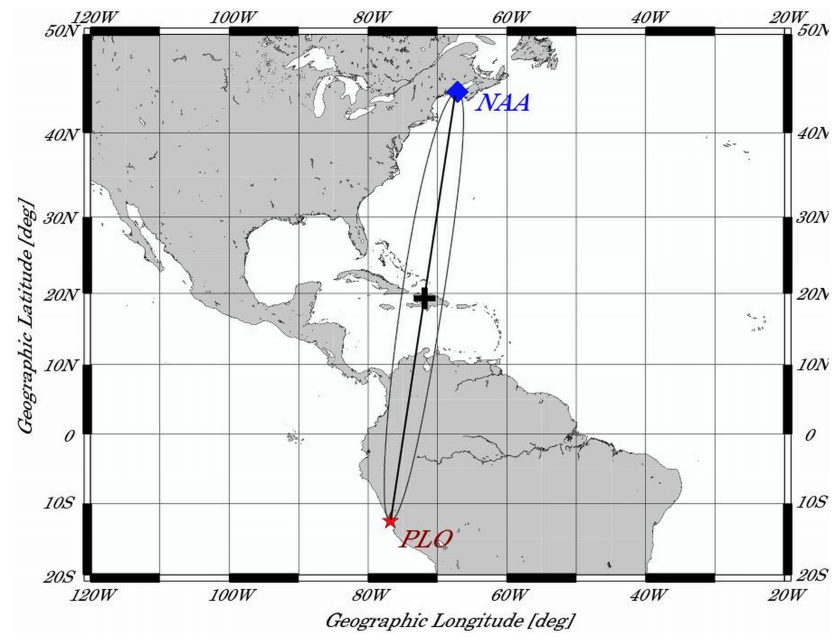

Fig. 1. Relative location of the VLF transmitter (NAA), a VLF receiving station in Peru (PLO) and the EQ epicentre (indicated by a cross). The great circle path and the fifth Fresnel zone are also indicated.

same period of a few days. From the periods satisfying this criterion, the first one corresponds to the middle of December 2009 and the second is just around New Year's day, 2010. The third one is just after the main shock. The last one is nearly at the end of our analysis period at the end of March 2010, which will be discussed later. The 2nd period just around New Year's day is likely considered to be a clear precursor to the 2010 Haiti EQ, because the time lag of about 12 days is very consistent with the result by both previous case studies (e.g., Hayakawa, 2007, 2009) and statistical studies (Hayakawa et al., 2010a). As for this period, the most important quantity, the trend, appears to be very much depleted exceeding a $2 \sigma$ level. The 2 nd parameter, dispersion is seen to increase and approach the $2 \sigma$ line and the $3 \mathrm{rd}$ parameter of NF is found to be enhanced exceeding the $2 \sigma$ criterion. Therefore, this anomaly is highly likely to be a precursor to the Haiti EQ. A few days just after the main shock of 12 January 2010 (this is the 3rd period), we observe another anomaly, in which we find a decrease in trend (close to but not exceeding the $2 \sigma$ line) and simultaneous increases in dispersion and NF. This might be an after-effect of the main shock.

We then pay attention to the additional periods of anomalous VLF/LF behaviours. One conspicuous anomaly around 12 December 2009, about one month before the main shock, is characterised by the simultaneous decrease in trend and increases in dispersion and NF, so that it is reasonable to regard this as being seismogenic, though we do not know in which way this anomaly is related to the Haiti EQ. Of course, it seems unrealistic to say that this anomaly is the conventional precursor to the main shock because previous studies (Hayakawa et al., 2010a, b) has indicated that the precursor appears one week to 10 days 


\section{$1 / 12$ Haichi $E Q$ \\ M7.010km}

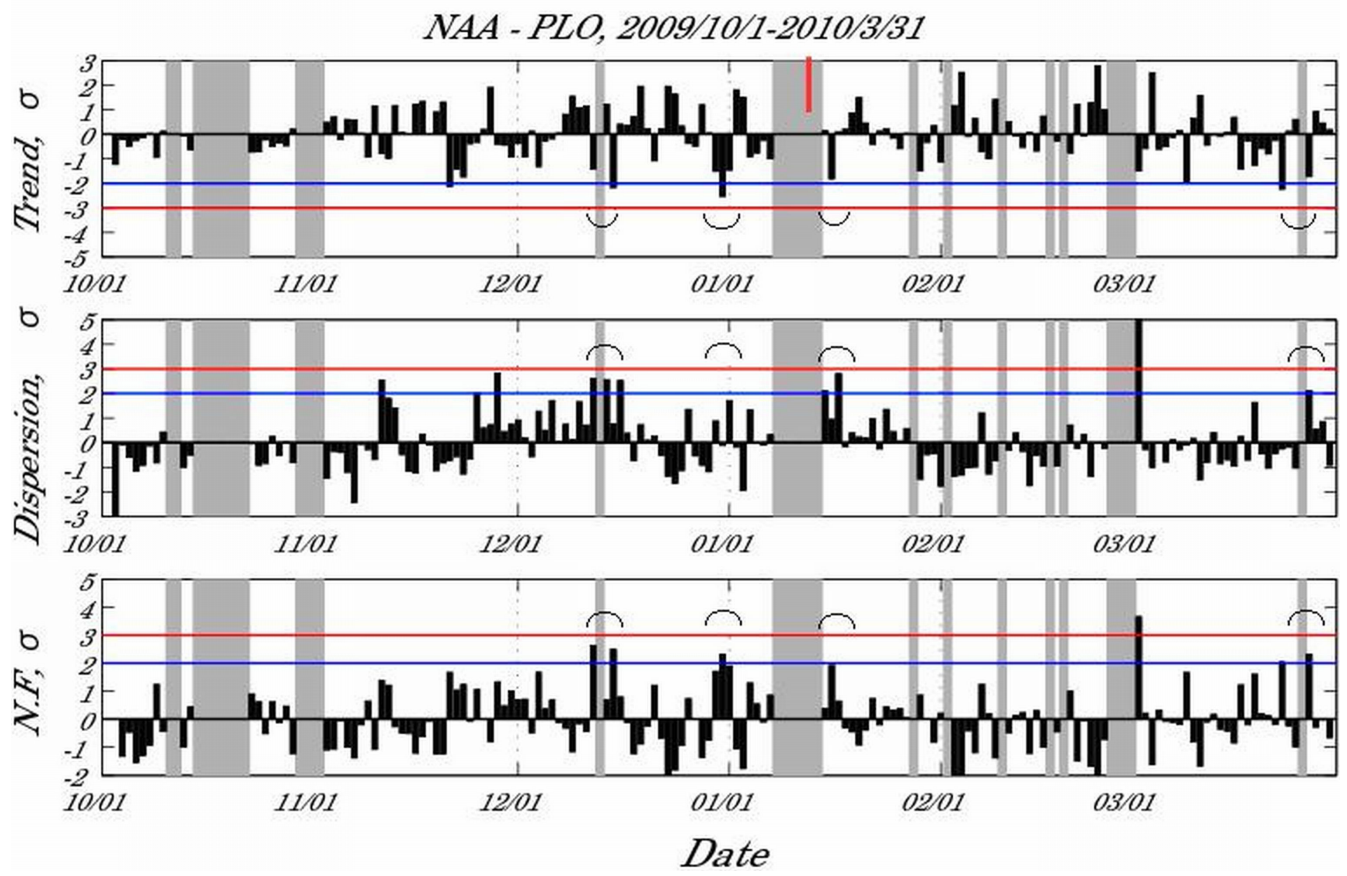

Fig. 2. Temporal evolutions of three physical parameters of VLF propagation characteristics (from the top, trend (mean nighttime amplitude), dispersion and NF (nighttime fluctuation)). All of these parameters are normalized with the corresponding standard deviation $(\sigma)$ before the current day ( -30 to -1 day of the current day). Grey areas indicate no observation. The date is from the beginning of 2009 to the end of March 2010, and EQ occurrence day is located approximately in the middle of our period. Of particular interest are the periods when the trend is decreased and the dispersion and NF show nearly simultaneous increases.

before an EQ. An additional, but less conspicuous, peak can be seen just around 12 November 2009, for which we see a certain decrease in trend and the simultaneous significant increases in dispersion and NF, again suggesting a seismogenic effect. We have to think of a possibility to consider these two anomalous time periods with seismogenic property in a way consistent with the main shock of the Haiti EQ. One possible way might be the effect of the Earth's tides discussed in Tanaka et al. (2004), who have shown a statistical evidence on the tidal effect in earthquake occurrence (the synodic month of 29.5 days). Then, Hayakawa et al. (2010c) have recently studied the effect of this Earth's tide even in seismogenic phenomena and this effect is found to be evident especially in the lithospheric phenomenon like seismogenic ULF emissions, and also in the seismo-ionospheric perturbations. The temporal spacing among 12 November, $\sim 12$ December and the main shock on 12 January, is found to be approximately one month, which might then be attributed to be the effect of the Earth's tides. This kind of Earth's tides can be seen only in association with huge EQs (Tanaka et al., 2004), and in this sense the present 2010 Haiti EQ is such an example.

Finally, we discuss the last VLF anomaly on $\sim 20$ March 2010. Of course, this anomaly is not associated with any of the aftershocks of the Haiti EQ, so that we have looked at any other EQs taking place within or around the wave sensitive area. We can list the following three EQs: 3 January $(M=$ $5.7, d($ depth $\left.)=140 \mathrm{~km} ; 9.03^{\circ} \mathrm{S}, 78.05^{\circ} \mathrm{W}\right), 25$ January $\left(M=5.8, d=154 \mathrm{~km} ; 8.47^{\circ} \mathrm{S} 74.73^{\circ} \mathrm{W}\right)$ and 28 March $\left(M=5.3, d=56 \mathrm{~km} ; 10.57^{\circ} \mathrm{S}, 78.81^{\circ} \mathrm{W}\right)$. The first two EQs are too deep to induce any ionospheric perturbations, based on the previous results by Kasahara et al. (2010), but the last and shallow EQ on 28 March is a possible candidate to have induced a seismo-ionospheric perturbation, about eight days before the main shock. So, we can say that the last VLF anomaly around 20 March is likely to be a precursor of the EQ on 28 March.

\section{Summary and discussion}

The analysis of subionospheric propagation from the transmitter of NAA $(24 \mathrm{kHz})$ to a VLF receiving station at PLO in Peru has yielded the following findings for the 2010 Haiti EQ.

1. The nighttime fluctuation method based on the measurements of three physical parameters - (1) trend, 
(2) dispersion, and (3) NF - is found to be effective for a medium distance $(6.43 \mathrm{Mm})$ VLF propagation path.

2. The VLF anomaly, characterised by a significant decrease in trend and simultaneous enhancements of dispersion and NF, is found to be detected about 12 days before the main shock of the 2010 Haiti EQ.

3. Additional anomalies are observed around 12 November and 12 December 2009, which are likely to be a signature of the Earth's tides in the dataset used for the Haiti EQ.

As for point (1), the effectiveness of the nighttime fluctuation method to this Haiti EQ case is considered to be a further confirmation of our recent paper by Kasahara et al. (2010a) in which the propagation from the NWC transmitter (Australia) to Japanese stations $(6-8 \mathrm{Mm})$ is effectively utilized to investigate the EQ signatures in the middle of the propagation path (such as the Philippines region).

Then the propagation anomaly for this Haiti EQ is characterised by the significant decrease in trend, together with the simultaneous enhancements both in dispersion and NF - point (2), which seems to be consistent with the conclusion obtained by Kasahara et al. (2010). The behaviour of the VLF anomaly, described in this paper, is exactly the same as that found for short-distance (less than 2$3 \mathrm{Mm}$ ) propagations (Hayakawa et al., 2010a). The epicentre of the present EQ happened to be located approximately on the great-circle path well within the wave sensitive area, so that it is very reasonable and acceptable for us to anticipate such a clear VLF propagation anomaly. The lead time of the anomaly found for this Haiti case is about 12 days, which seems to be slightly larger than the usual value of one week reported in Hayakawa et al. (2010a), but is not an unfavourable value.

As it was mentioned as point (3), additional VLF propagation anomalies seem to be observed one month and two months before the main shock of the EQ. The effect of the Earth's tides was recently found to be also observed in seismo-electromagnetic phenomena as in Hayakawa et al. (2009). This 2010 Haiti EQ was large enough, with a magnitude of 7.0, to be associated with the effect of the Earth's tide.

Finally, an additional VLF anomaly has been found in association with a subsequent shallow EQ in the northern part of Peru about 8 days later.

This is a preliminary analysis result, and many things are still to be done. By using the network observation for different combinations of transmitters and receivers, we will analyse, in detail, the temporal changes and spatial scale and structure of the ionospheric perturbation associated with this 2010 Haiti EQ, which would provide much more information on the lithosphere-ionospheric coupling mechanism.
Acknowledgements. Two of the authors (JPR and FCPB) would like to thank funding agencies MACKPESQUISA, CNPq (Proc. 304433/2004-7, 490124/2006-2), FAPESP (Proc. 2006/02979-0, 2007/05630-1), and WCD thanks the support of CONIDA. Japanese colleagues thank NICT (National Institute of Information and Communications Technology), Japan for its financial support.

Edited by: T. Maggipinto

Reviewed by: P. F. Biagi and A. P. Nickolaenko

\section{References}

Biagi, P. F., Castellana, L., Maggipinto, T., Loiacono, D., Schiavulli, L., Ligonzo, T., Fiore, M., Suciu, E., and Ermini, A.: A pre seismic radio anomaly revealed in the area where the Abruzzo earthquake $(M=6.3)$ occurred on 6 April 2009, Nat. Hazards Earth Syst. Sci., 9, 1551-1556, doi:10.5194/nhess-91551-2009, 2009.

Chakrabarti, S. K., Sasmal, S., and Chakrabarti, S.: Ionospheric anomaly due to seismic activities - Part 2: Evidence from D-layer preparation and disappearance times, Nat. Hazards Earth Syst. Sci., 10, 1751-1757, doi:10.5194/nhess-10-1751-2010, 2010.

Hayakawa, M.: VLF/LF radio sounding of ionospheric perturbations associated with earthquakes, Sensors, 7, 1141-1158, 2007.

Hayakawa, M.: Lower ionospheric pertubations associated with erathquakes, as detected by subionospheric VLF/LF radio waves, in: Electromagnetic Phenomena Associated with Earthquakes, edited by: Hayakawa, M., Transworld Research Network, Trivandrum, India, chapter 6, 137-185, 2009.

Hayakawa, M. and Hobara, Y.: Current status of seismoelectromagnetics for short-term earthquake prediction, Geomatics, Natural Hazards and Risk, 1(2), 115-155, 2010.

Hayakawa, M., Molchanov, O. A., Ondoh, T., and Kawai, E.: The precursory signature effect of the Kobe earthquake on VLF subionospheric signals, J. Comm. Res. Lab., Tokyo, 43, 169180, 1996.

Hayakawa, M., Ohta, K., Nickolaenko, A. P., and Ando, Y.: Anomalous effect in Schumann resonance phenomena observed in Japan, possibly associated with the Chi-chi earthquake in Taiwan, Ann. Geophys., 23, 1335-1346, doi:10.5194/angeo-231335-2005, 2005.

Hayakawa, M., Ohta, K., Maekawa, S., Yamauchi, T., Ida, Y., Gotoh, T., Yonaiguchi, N., Sasaki, H., and Nakamura, T.: Electromagnetic precursors to the 2004 Mid Niigata Prefecture earthquake, Phys. Chem. Earth, 31, 356-364, 2006.

Hayakawa, M., Sue, Y., and Nakamura, T.: The effect of earth tides as observed in seismo-electromagnetic precursory signals, Nat. Hazards Earth Syst. Sci., 9, 1733-1741, doi:10.5194/nhess-91733-2009, 2009.

Hayakawa, M., Kasahara, Y., Nakamura, T., Hobara, Y., Rozhnoi, A., Solovieva, M., and Molchanov, O. A.: A statistical study on the correlation between lower ionospheric perturbations as seen by subionospheric VLF/LF propagation and earthquakes, J. Geophys. Res., 115, A09305, doi:10.1029/2009JA015143, 2010a.

Hayakawa, M., Kasahara, Y., Nakamura, T., Hobara, Y., Rozhnoi, A., Solovieva, M., and Molchanov, O. A.: On the corsolation between ionospheric perturbations as detected by subionospheric 
VLF/LF signals and earthquakes as characterized by seismic intensity, J. Atmos. Sol.-Terr. Phy., 72, 982-987, 2010b.

Horie, T., Maekawa, S., Yamauchi, T., and Hayakawa, M.: A possible effect of ionospheric perturbations associated with the Sumatra earthquake, as revealed from subionospheric very-lowfrequency (VLF) propagation (NWC-Japan), Int. J. Remote Sens., 28(13), 3133-3139, 2007a.

Horie, T., Yamauchi, T., Yoshida, M., and Hayakawa, M.: The wave-like structures of ionospheric perturbation associated with Sumatra earthquake of 26 December 2004, as revealed from VLF observation in Japan of NWC signals, J. Atmos. Sol.-Terr. Phy., 69, 1021-1028, 2007b.

Kasahara, Y., Muto, F., Horie, T., Yoshida, M., Hayakawa, M., Ohta, K., Rozhnoi, A., Solovieva, M., and Molchanov, O. A.: On the statistical correlation between the ionospheric perturbations as detected by subionospheric VLF/LF propagation anomalies and earthquakes, Nat. Hazards Earth Syst. Sci., 8, 653-656, doi:10.5194/nhess-8-653-2008, 2008.

Kasahara, Y., Muto, F., Hobara, Y., and Hayakawa, M.: The ionospheric perturbations associated with Asian earthquakes as seen from the subionospheric propagation from NWC to Japanese stations, Nat. Hazards Earth Syst. Sci., 10, 581-588, doi:10.5194/nhess-10-581-2010, 2010.

Maekawa, S. and Hayakawa, M.: A statistical study on the dependence of characteristics of VLF/LF terminator, IEEJ Transactions on Fundamentals and Materials, 126(4), 220-226, 2006.

Maekawa, S., Horie, T., Yamauchi, T., Sawaya, T., Ishikawa, M., Hayakawa, M., and Sasaki, H.: A statistical study on the effect of earthquakes on the ionosphere, based on the subionospheric LF propagation data in Japan, Ann. Geophys., 24, 2219-2225, doi:10.5194/angeo-24-2219-2006, 2006.

Molchanov, O. A., Hayakawa, M., Ondoh, T., and Kawai, E.: Precursory effects in the subionospheric VLF signals for the Kobe earthquake, Phys. Earth Planet. In., 105, 239-248, 1998.
Molchanov, O., Rozhnoi, A., Solovieva, M., Akentieva, O., Berthelier, J. J., Parrot, M., Lefeuvre, F., Biagi, P. F., Castellana, L., and Hayakawa, M.: Global diagnostics of the ionospheric perturbations related to the seismic activity using the VLF radio signals collected on the DEMETER satellite, Nat. Hazards Earth Syst. Sci., 6, 745-753, doi:10.5194/nhess-6-745-2006, 2006.

Ohta, K., K., Umeda, N., Watanabe, and Hayakawa, M.: Relationship between ELF magnetic fields and Taiwan earthquake, in: Seismo Electromagnetics (Lithosphere-Atmosphere-Ionosphere Coupling), edited by: Hayakawa, M., and Molchanov, O. A., TERRAPUB, Tokyo, 233-237, 2002

Raulin, J. P., David, P., Hadano, R., Saraiva, A. C. V., Correia, E., and Kaufman, P.: The South America VLF NETwork (SAVNET), Earth Moon Planets, 104, 247-261, 2009.

Rozhnoi, A., Solovieva, M. S., Molchanov, O. A., and Hayakawa, M.: Middle latitude $\mathrm{LF}(40 \mathrm{kHz})$ phase variations associated with earthquakes for quiet and disturbed geomagnetic conditions, Phys. Chem. Earth, 29, 589-598, 2004.

Rozhnoi, A., Solovieva, M., Molchanov, O., Schwingenschuh, K., Boudjada, M., Biagi, P. F., Maggipinto, T., Castellana, L., Ermini, A., and Hayakawa, M.: Anomalies in VLF radio signals prior the Abruzzo earthquake $(M=6.3)$ on 6 April 2009, Nat. Hazards Earth Syst. Sci., 9, 1727-1732, doi:10.5194/nhess-91727-2009, 2009.

Shvets, A. V., Hayakawa, M., and Maekawa, S.: Results of subionospheric radio LF monitoring prior to the Tokachi $(M=8$, Hokkaido, 25 September 2003) earthquake, Nat. Hazards Earth Syst. Sci., 4, 647-653, doi:10.5194/nhess-4-647-2004, 2004.

Tanaka, S., Ohtake, M., and Sato, H.: Tidal triggering of earthquakes in Japan related to the regional tectonic stress, Earth Planets Space, 56(5), 511-515, 2004.

Yamauchi, T., Maekawa, S., Horie, T., Hayakawa, M., and Soloviev, O.: Subionospheric VLF/LF monitoring of ionospheric perturbations for the 2004 Mid-Niigata earthquake and their structure and dynamics, J. Atmos. Sol.-Terr. Phy., 69, 793-802, 2007. 